CERN-TH/95-321

ENSLAPP-A-565/95

SNUTP 95-119

hep-th/9512030

\title{
Lagrangian Formulation of Symmetric Space sine-Gordon Models
}

\author{
Ioannis Bakas 周 \\ Theory Division, CERN, 1211 Geneva 23, Switzerland, and \\ Laboratoire de Physique Theorique ENSLAPP, 74941 Annecy-le-Vieux, Franç \\ Q-Han Park and Hyun-Jong Shin"T \\ Department of Physics, and Research Institute of Basic Sciences \\ Kyunghee University, Seoul, 130-701, Korea
}

\begin{abstract}
The symmetric space sine-Gordon models arise by conformal reduction of ordinary 2-dim $\sigma$-models, and they are integrable exhibiting a black-hole type metric in target space. We provide a Lagrangian formulation of these systems by considering a triplet of Lie groups $F \supset G \supset H$. We show that for every symmetric space $F / G$, the generalized sine-Gordon models can be derived from the $G / H$ WZW action, plus a potential term that is algebraically specified. Thus, the symmetric space sine-Gordon models describe certain integrable perturbations of coset conformal field theories at the classical level. We also briefly discuss their vacuum structure, Backlund transformations, and soliton solutions.
\end{abstract}

December 1995

\footnotetext{
*Permanent address: Department of Physics, University of Patras, 26110 Patras, Greece

${ }^{\dagger}$ e-mail address: BAKAS@SURYA11.CERN.CH, BAKAS@LAPPHP8.IN2P3.FR

${ }^{\ddagger}$ Present address

§e-mail address: QPARK@NMS.KYUNGHEE.AC.KR

๑e-mail address: HJSHIN@NMS.KYUNGHEE.AC.KR
} 


\section{Introduction}

Ordinary 2-dim $\sigma$-models were intensively investigated in the past and their integrability properties are very well established (see for instance [1], and references therein). These models are classically conformal invariant, and therefore the freedom to choose coordinates can be exploited setting $T_{z z}=T_{\bar{z} \bar{z}}=1$ for the components of the classical stress-energy tensor. This choice was first implemented by Pohlmeyer to the equations of motion, leading to another class of integrable systems known as reduced $\sigma$-models [2]. An alternative description was given by Lund and Regge thinking of the classical equations of motion as describing the embedding of a 2-dim surface in the target space of the $\sigma$-model, which in turn is embedded in flat space [3]. Then, the reduction procedure is analogous to choosing the orthonormal gauge in bosonic string theory, and the reduced $\sigma$-model describes the dynamics of the transverse degrees of freedom after solving the constraints. f The simplest example is the reduction of the $S^{2}$ non-linear $\sigma$-model, which yields the celebrated sine-Gordon equation. This method has been generalized to $F / G$ $\sigma$-models following a systematic group theoretical approach that leads to the so called symmetric space sine-Gordon models (SSSG) [4] [5] [6].

The first non-trivial example of such a multi-component generalization is given by the complex sine-Gordon model, which is obtained from the $S^{3} \simeq S O(4) / S O(3) \sigma$-model by reduction. It involves two target space fields $\alpha$ and $\beta$ with Lagrangian

$$
\mathcal{L}=\partial \alpha \bar{\partial} \alpha+\cot ^{2} \alpha \partial \beta \bar{\partial} \beta+\cos ^{2} \alpha
$$

Another interesting example of SSSG that arises as reduced $C P^{2} \simeq S U(3) / U(2) \sigma$-model involves three fields $\alpha, \beta$ and $\gamma$ with Lagrangian

$$
\mathcal{L}=\partial \alpha \bar{\partial} \alpha+\frac{1}{4} \partial \beta \bar{\partial} \beta+\cot ^{2} \alpha \partial \gamma \bar{\partial} \gamma+2 \cos \alpha \cos (\beta-\gamma)
$$

All other SSSG models have a well established Lax-pair formulation, according to the general group theoretical scheme for reducing the classical equations of motion of 2-dim $\sigma$-models [4] [5], but there has been no Lagrangian formulation known for them up to this day. The main difficulty is to find a general parametrization of the target space fields in terms of the Lax-pair variables, so that a Lagrangian formulation becomes possible. In the known examples this is described by non-local transformation of variables whose exact form has not yet been found in the general $F / G$ case.

Our interest in the subject originates from the possible interpretation of (1.1) and (1.2) as integrable perturbations of conformal field theory (CFT) coset models. The reduction procedure, which spoils conformal invariance while preserving integrability, has a rather dramatic effect in the target space structure of $\sigma$-models. It is intriguing that the target space metric of the above two SSSG models is very different from ordinary $\sigma$-models in

*Of course, ordinary $\sigma$-models are not consistent string backgrounds quantum mechanically; this geometrical approach is only used to motivate the definition of classical reduced $\sigma$-models, as it was originally done in the literature. 
that there are singularities reminiscent of CFT black-hole backgrounds in string theory (although the black-hole interpretation is more appropriate for non-compact cosets [7]). The complex sine-Gordon model (1.1) has already been considered in some detail [8] [9], providing the Lagrangian formulation of the parafermionic $S U(2) / U(1)$ coset model perturbed by its first thermal operator. We will see later that the reduced $C P^{2}$ model can also be reformulated as a perturbed $S U(2) \times U(1) / U(1)$ CFT coset. It will be clear from our description that the non-local field redefinitions, used in the past to obtain (1.1) and (1.2) from the corresponding SSSG Lax-pairs, provide the classical parafermion variables of these CFT cosets.

In this paper we present a systematic Lagrangian formulation of the SSSG models for symmetric spaces $F / G$, using the gauged WZW action for $G / H$ plus a suitable deforming potential term. The general scheme for choosing the triplet of Lie algebras $(\mathbf{f}, \mathbf{g}, \mathbf{h})$ with respective associated groups $F \supset G \supset H$ will be explained in detail. Our construction shows that all SSSG models correspond to integrable deformations of certain conformal field theories, although further work is required to identify the quantum operators associated with the potential term in all different cases. This approach certainly serves as a basis for having a completely new look at the quantum structure of the various multi-component sine-Gordon models. We apply our approach to the symmetric spaces $F / G \simeq S O(n+1) / S O(n), S U(n) / S O(n), S U(n+1) / U(n)$, and rederive the Lax-pair formulation of the SSSG models from the WZW point of view. We also construct a new class of models based on the symmetric space $S p(n) / U(n)$. Finally, we consider the form of Backlund transformations, and present as example the 1-soliton solution of the $S U(3) / S O(3)$ SSSG model. Further generalizations of our scheme are also briefly discussed.

We note that for all SSSG models other than (1.1) and (1.2), the corresponding $G / H$ WZW cosets turn out to have non-abelian group $H$. It will be clear from our presentation that the required non-local field redefinitions for having a Lagrangian description of the underlying Lax-pair equations are actually equivalent to introducing non-abelian parafermions in the general case, as non-local functions of the target space fields. It might explain why this problem was not solved fifteen years ago, without the CFT interpretation of these models.

\section{The general scheme}

Let $F / G$ be a symmetric space, where the Lie algebra decomposition $\mathbf{f}=\mathbf{g} \oplus \mathbf{k}$ satisfies the commutation relations

$$
[\mathrm{g}, \mathrm{g}] \subset \mathbf{g},[\mathrm{g}, \mathrm{k}] \subset \mathbf{k},[\mathrm{k}, \mathrm{k}] \subset \mathbf{g} .
$$

We take two arbitrary elements $T, \bar{T}$ of $\mathbf{k}$ and define $\mathbf{h}$ as the simultaneous centralizer of $T$ and $\bar{T}$, i.e. $\mathbf{h}=C_{\mathbf{g}}(T, \bar{T})=\{R \in \mathbf{g}:[R, T]=0=[R, \bar{T}]\}$. Then, the Lagrangian formulation of the SSSG model for the symmetric space $F / G$ is given by the gauged $G / H$ 
WZW action plus a potential term,

$$
I=I_{W Z W}(g)+\frac{1}{2 \pi} \int \operatorname{Tr}\left(-A \bar{\partial} g g^{-1}+\bar{A} g^{-1} \partial g+A g \bar{A} g^{-1}-A \bar{A}\right)-I_{P}(g, T, \bar{T})
$$

where $I_{W Z W}(g)$ is the WZW action for a map $g: M \rightarrow G \subset F$ of a Lie group $G$ defined on two-dimensional Minkowski space $M$ 10. The connections $A, \bar{A}$ gauge the diagonal subgroup $H$ of $G$. The potential term is given in terms of $T$ and $\bar{T}$,

$$
I_{P}(g, T, \bar{T})=\frac{m^{2}}{2 \pi} \int \operatorname{Tr}\left(g T g^{-1} \bar{T}\right)
$$

where $m^{2}$ is a mass parameter.

The action (2.2) without the potential term is precisely the action for a conformal field theory based on the coset $G / H$ [11]. Therefore, our model describes an integrable perturbation of this coset conformal field theory with a deforming potential term that is characterized by $T$ and $\bar{T}$ associated with the embedding of $G$ into $F$. In order to understand the integrability properties of the model, and make precise connection with the usual formulation of the SSSG models, we should write the equations of motion in a zero cuvature form. Varying the action with respect to $g$ we have

$$
\delta_{g} I=\frac{1}{2 \pi} \int \operatorname{Tr}\left(-\left[\bar{\partial}+\bar{A}, \partial+g^{-1} \partial g+g^{-1} A g\right]+m^{2}\left[T, g^{-1} \bar{T} g\right]\right) g^{-1} \delta g=0 .
$$

Since $A$ and $\bar{A}$ commute with $T$ and $\bar{T}$ we have an identity $[\bar{\partial}+\bar{A}, T]=0$, and also

$$
\partial\left(g^{-1} \bar{T} g\right)+\left[g^{-1} \partial g+g^{-1} A g, g^{-1} \bar{T} g\right]=0
$$

which can be combined with (2.4) to give the equivalent zero cuvature expression

$$
\left[\partial+g^{-1} \partial g+g^{-1} A g+\lambda T, \bar{\partial}+\bar{A}+\frac{m^{2}}{\lambda} g^{-1} \bar{T} g\right]=0
$$

where $\lambda$ is an arbitrary spectral parameter. This last equation arises as the integrability condition of the linear system

$$
\left(\partial+g^{-1} \partial g+g^{-1} A g+\lambda T\right) \Psi=0 \quad, \quad\left(\bar{\partial}+\bar{A}+\frac{m^{2}}{\lambda} g^{-1} \bar{T} g\right) \Psi=0 .
$$

Note that the linear system (2.7) is written utilizing the full algebra $\mathbf{f}$, whereas the integrability equation (2.6) is only restricted to the subalgebra g due to the commutation relations $(2.1)$.

The constraint equations arising from the $A$ and $\bar{A}$ variations are

$$
\begin{aligned}
& \delta_{A} I=\frac{1}{2 \pi} \int \operatorname{Tr}\left(-\bar{\partial} g g^{-1}+g \bar{A} g^{-1}-\bar{A}\right) \delta A=0 \\
& \delta_{\bar{A}} I=\frac{1}{2 \pi} \int \operatorname{Tr}\left(g^{-1} \partial g+g^{-1} A g-A\right) \delta \bar{A}=0
\end{aligned}
$$


which, when combined with (2.6), yield the zero curvature condition $[\partial+A, \bar{\partial}+\bar{A}]=0$. This allows us to fix the gauge, and without loss of generality we set $A=\bar{A}=0$ from now on. Then, (2.6) becomes

$$
\bar{\partial}\left(g^{-1} \partial g\right)-m^{2}\left[T, g^{-1} \bar{T} g\right]=0,
$$

and the constraint equations reduce to

$$
\left(g^{-1} \partial g\right)_{\mathbf{h}}=0, \quad\left(\bar{\partial} g g^{-1}\right)_{\mathbf{h}}=0,
$$

where the subscript $\mathbf{h}$ denotes the projection to the $\mathbf{h}$ subalgebra.

We may solve the equations (2.5) and (2.11) introducing explicit parametrizations of $g^{-1} \bar{T} g$, so that the equation of motion for $g^{-1} \partial g$, (2.10), becomes a vector type generalization of the usual sine-Gordon equation. This is actually done in the next section for various choices of symmetric spaces. In fact, only these vector type equations and their zero curvature expressions are used to define the SSSG model, as in the earlier works [4] [5]. We emphasize, however, that even though the equations of motion can be expressed solely in terms of the $g^{-1} \bar{T} g$ variables, the Lagrangian formulation requires a full parametrization of $g$. The number of parameters of $g$ is bigger than $g^{-1} \bar{T} g$, and the constraints we have imposed match the difference.

\section{$3 \quad$ Symmetric spaces}

Compact symmetric spaces have been completely classified by Cartan (see for instance [12]), and they are labeled by type I and type II. Here, we consider type I symmetric spaces and present explicit equations for most of them. At the end of the paper, we will make some comments on the type II spaces, and make connections with other examples of generalized sine-Gordon models based on $S L(2)$ embeddings [13].

$$
\text { I. } F / G=S O(n+1) / S O(n)
$$

We choose $T, \bar{T}$ and the embedding of $S O(n)$ into $S O(n+1)$ as follows,

$$
T=\bar{T}=\left(\begin{array}{ccccc}
0 & -1 & 0 & \ldots & 0 \\
1 & 0 & 0 & \ldots & 0 \\
0 & 0 & 0 & \ldots & 0 \\
\vdots & & & & \vdots \\
0 & & \ldots & & 0
\end{array}\right), \quad \tilde{g}=\left(\begin{array}{cccc}
1 & 0 & \ldots & 0 \\
0 & & & \\
\vdots & &
\end{array}\right) \in S O S O(n) \quad(n+1)
$$

so that the stability group $H=S O(n-1)$. We also introduce explicit parametrizations,

$$
g^{-1} \bar{T} g=\left(\begin{array}{cccc}
0 & V_{0} & \cdots & V_{n-1} \\
-V_{0} & 0 & \cdots & 0 \\
\vdots & \vdots & & \vdots \\
-V_{n-1} & 0 & \cdots & 0
\end{array}\right), \quad g^{-1} \partial g=\left(\begin{array}{ccccc}
0 & 0 & 0 & \cdots & 0 \\
0 & 0 & E_{1} & \cdots & E_{n-1} \\
0 & -E_{1} & & & \\
\vdots & \vdots & & A=0 & \\
0 & -E_{n-1} & & &
\end{array}\right)
$$


where we have imposed the constraint (2.11). Then, the elements $V_{i}=-g_{1, i+1}$, where $i=0,1, \ldots, n-1$, satisfy the normalization condition

$$
V_{0}^{2}+\sum_{k=1}^{n-1} V_{k}^{2}=\sum_{k=1}^{n} g_{1 k}^{2}=1
$$

The identity (2.5) resolves into component equations,

$$
\partial V_{0}+E_{k} V_{k}=0, \quad \partial V_{i}-V_{0} E_{i}=0 ; \quad i=1,2, \ldots, n-1,
$$

which can be solved for $E_{i}$,

$$
E_{i}=\frac{\partial V_{i}}{\sqrt{1-V_{k} V_{k}}}
$$

Then, (2.10) yields the vector type SSSG equation

$$
\bar{\partial} E_{i}-m^{2} V_{i} \equiv \bar{\partial} \frac{\partial V_{i}}{\sqrt{1-V_{k} V_{k}}}-m^{2} V_{i}=0,
$$

which reproduces the $S O(n+1) / S O(n)$ SSSG model [4] [5] from the $S O(n) / S O(n-1)$ gauged WZW theory.

II. $F / G=S U(n) / S O(n)$

Here, we choose $T, \bar{T}$

$$
T=\bar{T}=\left(\begin{array}{cccc}
-n+1 & 0 & \cdots & 0 \\
0 & 1 & & 0 \\
\vdots & & \ddots & \\
0 & 0 & & 1
\end{array}\right)
$$

and embed the $S O(n)$ group by restricting the $S U(n)$ elements to be real, so that the stability group $H=S O(n-1)$. We also introduce the parametrizations,

$g^{-1} \bar{T} g=1-n\left(\begin{array}{cccc}V_{0} & V_{1} & \ldots & V_{n-1} \\ V_{1} & & & \\ \vdots & & \frac{1}{V_{0}} V_{i} V_{j} & \\ V_{n-1} & & & \end{array}\right), g^{-1} \partial g=\left(\begin{array}{cccc}0 & E_{1} & \cdots & E_{n-1} \\ -E_{1} & & & \\ \vdots & & A=0 & \\ -E_{n-1} & & & \end{array}\right)$,

where $i, j$ run from 1 to $n-1$, and $V_{i}=g_{1,1} g_{1, i+1}$ satisfy the relation

$$
V_{0}^{2}+\sum_{k=1}^{n-1} V_{k}^{2}=V_{0}
$$

The identity (2.5) becomes

$$
\partial V_{0}+2 E_{k} V_{k}=0, \quad \partial V_{i}+\frac{1}{V_{0}} V_{i} V_{k} E_{k}-E_{i} V_{0}=0
$$


which can be solved for $E_{i}$,

$$
E_{i}=\frac{1}{\sqrt{V_{0}}} \partial\left(V_{i} \sqrt{V_{0}}\right)
$$

Changing variables, $V_{0}=1-U_{k} U_{k}$ and $V_{i}=\sqrt{1-U_{k} U_{k}} U_{i}$, we find that $(2.10)$ becomes the vector type sine-Gordon equation

$$
\bar{\partial} \frac{\partial U_{i}}{\sqrt{1-U_{k} U_{k}}}-m^{2} n^{2} \sqrt{1-U_{k} U_{k}} U_{i}=0 ; \quad i=1,2, \ldots, n-1
$$

for the $S U(n) / S O(n)$ SSSG model. This corresponds to the maximally degenerate case of the SSSG models in [5]. The generalization to nondegenerate cases is straightforward choosing $T=\bar{T}$ with distinct diagonal elements.

III. $F / G=S U(n+1) / U(n)$

We choose $T, \bar{T}$ and the embedding of the $U(n)$ group into $S U(n+1)$ as follows,

$$
T=\bar{T}=\left(\begin{array}{ccccc}
0 & -1 & 0 & \ldots & 0 \\
1 & 0 & 0 & \ldots & 0 \\
0 & 0 & 0 & \ldots & 0 \\
\vdots & & & & \vdots \\
0 & & \ldots & & 0
\end{array}\right), \quad \tilde{g}=\left(\begin{array}{cccc}
(\operatorname{det} g)^{-1} & 0 & \ldots & 0 \\
0 & & & \\
\vdots & & g \in U(n) \\
0 & & &
\end{array}\right) \in S U(n+1)
$$

so that $H=U(n-1)$. We also have

$$
g^{-1} \bar{T} g=\left(\begin{array}{cccc}
0 & -V_{0} & \cdots & V_{n-1} \\
V_{0}^{*} & 0 & \cdots & 0 \\
\vdots & \vdots & & \vdots \\
V_{n-1}^{*} & 0 & \cdots & 0
\end{array}\right), g^{-1} \partial g=\left(\begin{array}{ccccc}
i E_{0} & 0 & 0 & \cdots & 0 \\
0 & -i E_{0} & -E_{1} & \cdots & -E_{n-1} \\
0 & E_{1}^{*} & & & \\
\vdots & \vdots & & A=0 & \\
0 & E_{n-1}^{*} & &
\end{array}\right)
$$

satisfying the normalization condition

$$
V_{0} V_{0}^{*}+V_{k} V_{k}^{*}=1
$$

so that $V_{0}=e^{i \theta} \sqrt{1-V_{k} V_{k}^{*}}$ for some $\theta$. The identity (2.5) resolves into component equations

$$
\partial V_{i}+i E_{0} V_{i}+E_{i} V_{0}=0, \quad \partial V_{0}+2 i E_{0} V_{0}-V_{k} E_{k}^{*}=0,
$$

which can be solved for $E_{i}$,

$$
\begin{aligned}
E_{0} & =\frac{i}{6 V_{k} V_{k}^{*}-4}\left[V_{i}^{*} \partial V_{i}-\partial V_{i}^{*} V_{i}+2 i\left(1-V_{k} V_{k}^{*}\right) \partial \theta\right] \\
E_{i} & =-\frac{e^{-i \theta}}{\sqrt{1-V_{k} V_{k}^{*}}}\left(\partial V_{i}+i E_{0} V_{i}\right)
\end{aligned}
$$


Then, the corresponding SSSG equations become

$$
\begin{aligned}
\bar{\partial} E_{i}-m^{2} V_{i} & =0, \\
\bar{\partial} E_{0}-2 m^{2} \sin \theta \sqrt{1-V_{k} V_{k}^{*}} & =0
\end{aligned}
$$

reproducing the previously known result [4] [5] from the gauged $U(n) / U(n-1)$ WZW point of view.

IV. $F / G=S p(n) / U(n)$

In this case, which has not been considered before, we choose

$$
T=\bar{T}=\left(\begin{array}{cccc}
j & 0 & \cdots & 0 \\
0 & 0 & \cdots & 0 \\
\vdots & \vdots & & \vdots \\
0 & 0 & \cdots & 0
\end{array}\right),
$$

where $j$ is a quarternion satisfying the defining relations $i^{2}=j^{2}=k^{2}=-1, i j=-j i=$ $k, j k=-k j=i, k i=-i k=j$. We embed $U(n)$ into $S p(n)$ by restricting the $S p(n)$ elements to be complex. Then, the stability group $H=U(n-1)$ and

$$
g^{-1} \bar{T} g=j\left(\begin{array}{cccc}
V_{0}^{2} & V_{0} V_{1} & \cdots & V_{0} V_{n-1} \\
\vdots & \vdots & & \vdots \\
V_{n-1} V_{0} & V_{n-1} V_{1} & \cdots & V_{n-1}^{2}
\end{array}\right), g^{-1} \partial g=\left(\begin{array}{cccc}
i E_{0} & -E_{1} & \cdots & -E_{n-1} \\
E_{1}^{*} & & \\
\vdots & & A=0 \\
E_{n-1}^{*} &
\end{array}\right),
$$

where $V_{i}=g_{1, i+1}$ satisfy $V_{0} V_{0}^{*}+V_{k} V_{k}^{*}=1$, so that $V_{0}=e^{i \theta} \sqrt{1-V_{k} V_{k}^{*}}$ for some $\theta$. The identity (2.5) gives

$$
\begin{aligned}
\partial V_{i}+V_{0} E_{i} & =0 \\
\partial V_{0}-i E_{0} V_{0}-E_{k}^{*} V_{k} & =0
\end{aligned}
$$

which can be solved for $E_{0}, E_{i}$,

$$
\begin{aligned}
& E_{0}=\frac{-i}{2-2 V_{k} V_{k}^{*}}\left[2 i\left(1-V_{k} V_{k}^{*}\right) \partial \theta+V_{k} \partial V_{k}^{*}-\partial V_{k} V_{k}^{*}\right] \\
& E_{i}=-\frac{e^{-i \theta}}{\sqrt{1-V_{k} V_{k}^{*}}} \partial V_{i} .
\end{aligned}
$$

Then, the $U(n) / U(n-1)$ WZW model yields the $S p(n) / U(n)$ SSSG equations,

$$
\begin{aligned}
\bar{\partial} E_{0}+2 m^{2} \sin 2 \theta\left(1-V_{k} V_{k}^{*}\right) & =0, \\
\bar{\partial} E_{i}-m^{2} e^{i \theta} \sqrt{1-V_{k} V_{k}^{*}} V_{i} & =0,
\end{aligned}
$$

which clearly differ from case III above, due to the difference in embedding $U(n)$ in $F$. 


\section{Soliton solutions}

Since $T$ and $\bar{T}$ commute with $H$, the potential term is also invariant under the vector gauge symmetry of the gauged WZW action,

$$
g \rightarrow h^{-1} g h \quad, \quad A \rightarrow h^{-1} A h+h^{-1} \partial h \quad, \quad \bar{A} \rightarrow h^{-1} \bar{A} h+h^{-1} \bar{\partial} h
$$

for $h$ valued in $H$. This shows that the potential possesses a flat direction which is a mere gauge artifact and disappears after the gauge fixing. In fact, the connection components $A$ and $\bar{A}$, due to the lack of kinetic terms, play the role of Lagrange multipliers, which impose constraints that suppress the propagating degrees of freedom along the flat directions. However, there exists a true flat direction arising from the axial vector symmetry of the potential $I_{P}$,

$$
g \rightarrow h g h \quad, \quad A \rightarrow h A h+h \partial h \quad, \quad \bar{A} \rightarrow h \bar{A} h+h \bar{\partial} h,
$$

which in general is not a symmetry of the action (2.2). Thus, this axial vector symmetry results in a continuous degeneracy of the vacuum. On the other hand, due to the compactness of the group $G$, the vacuum of the theory also possesses discrete symmetries, which lead to the soliton solutions interpolating between two different vacua that are not connected by the flat directions. Such solutions may be constructed by applying the dressing method to the linear system of equations (2.7), or more directly using Backlund transformations.

The Backlund transformation for the symmetric space sine-Gordon models, in the gauge $A=\bar{A}=0$, is described by

$$
\Psi_{g}=\frac{\lambda}{\lambda-i \eta}\left(1+\frac{m^{2} \eta}{\lambda} g^{-1} M f\right) \Psi_{f},
$$

where $M$ is an arbitrary constant matrix satisfying the condition

$$
[\bar{T}, M]=0
$$

Here $f, g$ are elements of $G, \lambda$ is the spectral parameter, and $\eta \neq 0$ is a characteristic parameter of the solitons. $\Psi_{g}$ and $\Psi_{f}$ satisfy the the linear equations,

$$
\begin{array}{ll}
\left(\partial+g^{-1} \partial g+\lambda T\right) \Psi_{g}=0, & \left(\bar{\partial}+\frac{m^{2}}{\lambda} g^{-1} \bar{T} g\right) \Psi_{g}=0 \\
\left(\partial+f^{-1} \partial f+\lambda T\right) \Psi_{f}=0, & \left(\bar{\partial}+\frac{m^{2}}{\lambda} f^{-1} \bar{T} f\right) \Psi_{f}=0 .
\end{array}
$$

These equations allow us to eliminate $\Psi_{g}$ and $\Psi_{f}$ from (4.3), leading to the Backlund transformation written only in terms of $g$ and $f$,

$$
\begin{aligned}
g^{-1} \partial g-f^{-1} \partial f-m^{2} \eta\left[g^{-1} M f, T\right] & =0 \\
\eta \bar{\partial}\left(g^{-1} M f\right)+g^{-1} \bar{T} g-f^{-1} \bar{T} f & =0 .
\end{aligned}
$$


The Backlund transformation offers us the ability to calculate the 1-soliton solution of the theory starting from the vacuum solution, for which $f=1$, and multi-soliton solutions using non-abelian superposition rules [9].

Consider, for example, the 1-soliton solution of $S U(3) / S O(3)$. Specializing to $M^{-1} g=$ $g^{-1} M=2 P-1$, so that $P^{2}=P, P=P^{T}$, and setting $f=1$, we obtain from $(4.6)$

$$
2(2 P-1) \partial P+m^{2} \eta[T, 2 P-1]=0 .
$$

Multiplying with $(2 P-1)$ and subtracting the result from (4.8) we obtain

$$
(1-P)\left(\partial-m^{2} \eta T\right) P=0
$$

and similiarly from (4.7) we obtain

$$
(1-P)(\eta \bar{\partial}-\bar{T}) P=0 .
$$

To solve them, it is convenient to represent the projector $P$ in matrix form as $P_{i j}=s_{i} t_{j}$, where $i, j=1,2,3$, and impose the relation $\sum_{i} s_{i} t_{i}=1$. The property $P=P^{T}$ implies

$s_{i}=\alpha t_{i}$, where $\alpha=s_{1}^{2}+s_{2}^{2}+s_{3}^{2}$. Finally, using equations (4.9) and (4.10), we may write $s_{i}$ as follows, using $T=\bar{T}$ as in (3.7),

$$
s_{i}=\sum_{j}(\exp \Sigma T)_{i j} u_{j} ; \quad \Sigma=m^{2} \eta z+\frac{\bar{z}}{\eta},
$$

where $\left(u_{1}, u_{2}, u_{3}\right)$ are arbitrary constants parametrized as $(\cos \theta, \sin \theta \cos \phi, \sin \theta \sin \phi)$. Then, the Backlund transformation yields the 1-soliton solution of the equations of motion (3.12), which is summarized as follows,

$$
\begin{aligned}
E_{1} & =\left(g^{-1} \partial g\right)_{12}=2 m^{2} \eta(P T-T P)_{12}=\frac{3 m^{2} \eta \sin 2 \theta \cos \phi}{\cosh 3 \Sigma-\cos 2 \theta \sinh 3 \Sigma}, \\
V_{0} & =\left(g^{-1} \bar{T} g\right)_{11}=(\bar{T}-2 \eta \bar{\partial} P)_{11}=\left(\frac{-\sinh 3 \Sigma+\cos 2 \theta \cosh 3 \Sigma}{\cosh 3 \Sigma-\cos 2 \theta \sinh 3 \Sigma}\right)^{2}, \\
V_{1} & =\left(g^{-1} \bar{T} g\right)_{12}=\frac{-\sinh 3 \Sigma+\cos 2 \theta \cosh 3 \Sigma}{(\cosh 3 \Sigma-\cos 2 \theta \sinh 3 \Sigma)^{2}} \sin 2 \theta \cos \phi, \\
E_{2} & =E_{1} \tan \phi, \quad V_{2}=V_{1} \tan \phi .
\end{aligned}
$$

Other examples can be worked out in a similar way, but the computation becomes technically much more involved.

\section{Conclusions and further generalizations}

In this paper we have presented a systematic Lagrangian formulation of symmetric space sine-Gordon models in terms of the gauged WZW action, plus a deforming potential term that preserves the integrability of the system. Our construction is based on a triplet of 
Lie groups $(F, G, H)$, and it has been applied to certain classes of compact symmetric spaces of type I (in Cartan's classification). In our examples, $T$ and $\bar{T}$ were defined using the embedding of $G$ in $F$. However, the present framework is quite general and it encompasses other non-abelian generalizations of the sine-Gordon model as well. For instance, for compact symmetric spaces of type II, e.g. symmetric spaces of the form $G \times G / G$, the elements $g$ and $T$ take the form $g \otimes g$ and $T \otimes 1-1 \otimes T$ (and similarly for $\bar{T}$ ). The model then becomes effectively equivalent to (2.2), where $T$, $\bar{T}$ belong to the Lie algebra g. One such example is provided by the complex sine-Gordon model, which arises as reduced $S O(4) / S O(3) \simeq S O(3) \times S O(3) / S O(3) \sigma$-model; it has been described by the $S U(2) / U(1)$ WZW model with $T=\bar{T} \in U(1) \subset S U(2)$ [] [9].

Recently, there appeared a new class of generalized sine-Gordon models based on $S L(2)$ embeddings [13]. These systems were also constructed and classified according to a triplet of Lie groups $(F, G, H)$, where $G$ was chosen to be the zero graded part of $F$ in the $S L(2)$ embedding. If we identify $T$ and $\bar{T}$ with $J_{+}+J_{-}$of the embedded $S L(2)$ algebra, we find that all of these models (they are actually five different types) can be incorporated in our SSSG model construction with symmetric spaces $F / G$ corresponding to the triplets:

$A I I I \leftrightarrow(S U(2 n), S U(n) \times S U(n) \times U(1), S U(n))$,

$C I \leftrightarrow(S p(n), S U(n) \times U(1), S O(n))$,

$B D I \leftrightarrow(S O(n), S O(n-2) \times U(1), S O(n-3))$,

$D I I I \leftrightarrow(S O(4 n), S U(2 n) \times U(1), S p(n))$,

$E V I I \leftrightarrow\left(E_{7}, E_{6} \times U(1), H\right)$,

but we have been unable to determine $H$ in the last case. The interested reader should consult [13] for further understanding of the correspondences we are suggesting.

It is clear that the gauged WZW framework we have developed is the most general for describing various multi-component generalizations of the sine-Gordon model, using a deforming potential term $\operatorname{Tr}\left(g T g^{-1} \bar{T}\right)$ with appropriately chosen $T$ and $\bar{T}$ in each case. This suggests a perturbed conformal field theory approach to the quantization of these integrable systems, and therefore it is necessary for this purpose to identify correctly the CFT operators that correspond to the classical potential terms in the action. It will also be a useful exercise to find the general form of the non-local field redefinitions that were required in the old approaches for having a Lagrangian formulation of SSSG models. According to earlier work [8], they should involve the classical parafermion variables of the corresponding CFT cosets, taking into account appropriate non-abelian generalizations [14, and express them non-locally in terms of the target space fields. It will be also interesting to consider supersymmetric generalizations of SSSG models [5] in our context.

\section{ACKNOWLEDGEMENT}

We thank T. Hollowood and K. Sfetsos for useful discussions in the early stages of this 
work. I.B. is also grateful to the CTP of Seoul National University, the Physics Department of Kyunghee University, and the Asian-Pacific Center for Theoretical Physics for financial support and warm hospitality during his visit to Korea. Q.P. and H.J.S. are supported in part by the program of Basic Science Research, Ministry of Education BSRI-95-2442, and by Korea Science and Engineering Foundation through CTP/SNU.

\section{References}

[1] A. D'Adda, M. Luscher and P. DiVecchia, Phys. Rep. 49 (1979) 239; A. Perelomov, Phys. Rep. 146 (1987) 135.

[2] K. Pohlmeyer, Commun. Math. Phys. 46 (1976) 207.

[3] F. Lund and T. Regge, Phys. Rev. D14 (1976) 1524; F. Lund, Phys. Rev. Lett. 38 (1977) 1175; Ann. Phys. 115 (1978) 251.

[4] H. Eichenherr and K. Pohlmeyer, Phys. Lett. 89B (1979) 76; H. Eichenherr, Phys. Lett. 90B (1980) 121; H. Eichenherr and J. Honerkamp, J. Math. Phys. 22 (1981) 374.

[5] R. D'Auria, T. Regge and S. Sciuto, Phys. Lett. 89B (1980) 363; Nucl. Phys. 171B (1980) 167; R. D'Auria and S. Sciuto, Nucl. Phys. 171B (1980) 189.

[6] V.E. Zakharov and A.V. Mikhailov, Sov. Phys. JETP 47 (1978) 1017.

[7] E. Witten, Phys. Rev. D44 (1991) 314.

[8] I. Bakas, Int. J. Mod. Phys. A9 (1994) 3443.

[9] Q-H. Park, Phys. Lett. B328 (1994) 329; Q-H. Park and H.J. Shin, Phys. Lett. B347 (1995) 73; B359 (1995) 125.

[10] E. Witten, Commun. Math. Phys. 92 (1984) 455.

[11] D. Karabali, Q-H. Park, H.J. Schnitzer and Z. Yang, Phys. Lett. B216 (1989) 307; D. Karabali and H.J. Schnitzer, Nucl. Phys. B329 (1990) 649; K. Gawedski and A. Kupiainen, Phys. Lett. B215 (1988) 119; Nucl. Phys. B320 (1989) 625.

[12] S. Helgason, Differential geometry, Lie groups and symmetric spaces (Academic Press, New York, 1978).

[13] T.J. Hollowood, J.L. Miramontes and Q-H. Park, Nucl. Phys. B445 (1995) 451.

[14] K. Bardakci, M. Crescimanno and E. Rabinovici, Nucl. Phys. B344 (1990) 344; K. Bardakci, M. Crescimanno and S. Hotes, Nucl. Phys. B349 (1991) 439. 